\title{
SPATIAL ANALYSIS OF MULTI-CHANNEL EEG RECORDINGS THROUGH A FUZZY-RULE BASED SYSTEM IN THE DETECTION OF EPILEPTIFORM EVENTS
}

\author{
Christopher J. James ${ }^{1}$, Member, IEEE, Richard D. Jones ${ }^{2 *}$, Senior Member, IEEE, \\ Philip J. Bones ${ }^{1}$, Senior Member, IEEE, and Grant J. Carroll ${ }^{3}$ \\ ${ }^{1}$ Department of Electrical \& Electronic Engineering, University of Canterbury, \\ ${ }^{2}$ Department of Medical Physics \& Bioengineering, Christchurch Hospital, \\ ${ }^{3}$ Department of Neurology, Christchurch Hospital, \\ Christchurch, New Zealand
}

\begin{abstract}
A system has been developed which utilises fuzzy logic to perform spatial analysis of the multichannel EEG recording and forms the final stage of a multi-stage system to detect the presence of epileptiform events (EVs) in the EEG. This spatial-combiner consists of a set of 127 fuzzy-rules which define our expectation of the spatial distribution of an EV as measured across a 4channel bipolar chain of scalp electrodes. A set of probabilities assigned to each channel by the previous ANN-based stage of the EV-detection system are fuzzified into 5 fuzzy variables and the best matching fuzzy rule gives an output of either definite, probable or possible to indicate a detection of an EV on spatial grounds.

The system was tested on 8 clinical EEG recordings (7 epileptiform and 1 normal) which indicated a sensitivity of $55.3 \%$, a selectivity of $82.0 \%$ and a false detection rate of 7.2/hour. These results show a $\mathbf{5 0 - f o l d ~ d e c r e a s e ~ i n ~ t h e ~}$ false detection rate when compared to the performance of the system without spatial analysis, whilst maintaining a comparable level of sensitivity.
\end{abstract}

Keywords - fuzzy logic, spike detection, EEG, spatialanalysis.

\section{INTRODUCTION}

$\mathrm{D}$ ETECTING the presence of epileptiform discharges (EDs) in the electroencephalogram (EEG) is an important component in the diagnosis of epilepsy. EDs (or 'spikes') are manifest in the individual channels of the multichannel EEG recordings and together make up an epileptiform event (EV). Many methods have been developed for the automatic detection of EDs but all of these have, for the most part, met with limited success. The methods used have included mimetic methods [1], expert systems [2, 3], and artificial neural networks (ANNs) $[4,5]$.

*e-mail: r.jones@chmeds.ac.nz
More often than not, the detection methods are based on analysing single-channels of the multi-channel recordings independently - i.e., EDs are detected rather than EVs. This is somewhat surprising as it is well established that EEGers make considerable use of spatial contextual cues in the detection process. Some researchers have managed to incorporate spatial analysis in their detection systems. Webber et al. [5] performed detection based on 4 channels of EEG simultaneously being presented to a trained ANN whereas Glover et al. [3] and Dingle et al. [2] used expert systems to incorporate spatial contextual (and temporal) cues in the detection process, obtaining promising results $[2,3,6]$.

The method described here forms the final stage of a multi-stage system for the detection of EVs in the inter-ictal EEG and is called the spatial-combiner [7]. The spatialcombiner is used to perform spatial analysis on the outcome of the previous stages to transform a possible detection of an $\mathrm{ED}$ on a single channel basis to a detection of an EV using multi-channel cues. Similarly, possible false detections are avoided through the use of such spatial cues. The method is based on the use of fuzzy logic and allows the capture of the essence of the reasoning of an EEGer when performing spatial analysis.

\section{Methods}

\section{A. Fuzzy logic theory}

Fuzzy logic was first developed by Zadeh in 1965 [8] and is based on a mathematical theory which combines elements of multi-valued logic, probability theory, and artificial intelligence. Fuzzy logic simulates aspects of human thinking by incorporating the imprecision inherent in all physical systems [9]. In fuzzy systems, data is said to belong to fuzzy 'sets' and a degree of overlap between two or more sets is allowed, where a particular variable is allowed to be a member of one set with a particular degree of membership 
and a member in another, different, set with a different degree of membership. So a fuzzy set introduces vagueness (with the aim of reducing complexity) by eliminating the sharp boundary dividing members of the class from non-members. Membership functions define the degrees of membership between sets and the shape of the membership function can be any shape appropriate to the application; it is usually one of trapezoidal, sigmoidal or Gaussian. The linguistic variables (or fuzzy variables) used to describe fuzzy sets usually involve terms such as 'hot' or 'cold', 'high' or 'low', etc.

Rules are normally defined in the "IF condition THEN outcome" form, where the outcome of a rule depends on one or more conditions being met. For classical logic the conditions would consist of two-valued (crisp) conditions. Fuzzy logic offers the opportunity to perform some function based on a condition (or conditions) which allow degrees of membership [10]. An example of a fuzzy rule for a patient monitoring system, say, could be

\section{IF body_temperature is 'high' $\mathbf{O R}$ blood_pressure is 'low'}

\section{THEN}

$$
\text { alarm_level is 'high'. }
$$

When inputs are applied to the multiple rules of a fuzzy rulebase, all rules will respond with different membership values (albeit most may be 0 ). It then becomes necessary to combine the outcomes of all the rules and produce a single 'crisp' outcome for the input conditions given, this process is known as defuzzification. Many techniques are available for the defuzzification process but, in general, the two most common are to use the composite moment (i.e., centroid) or the composite maximum. The centroid takes the centre of gravity of the final fuzzy space and produces a result that is sensitive to all the rules, whereas the composite maximum produces a result that is sensitive to the single rule that has the highest outcome. Generally information based applications tend to use the composite maximum.

The block diagram of Fig. 1 depicts the fuzzy logic topology. The inputs are first fuzzified, inference then takes place on the fuzzy rule base, followed by defuzzification where the fuzzy outcome of the rules is converted to a crisp output.

In practice, a precise model of a biological system may not be known or it may be too difficult to model. In those cases, fuzzy logic may be an appropriate tool for modelling and controlling the biological system, since our knowledge and experience are directly contained and represented in the fuzzy logic model without the need for explicit mathematical models.

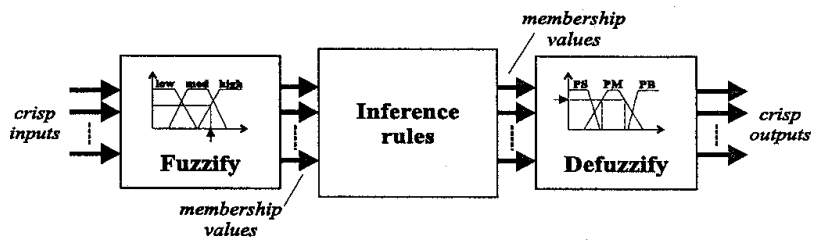

Fig. 1. A block diagram depicting the fuzzy logic topology.

\section{$B$. The spatial-combiner}

The spatial-combiner forms the last stage of a multi-stage spike detection system. The first two stages comprise a mimetic-based detector and a self-organising feature map (SOFM) ANN, both of which are described further in [7] and [11] (see Fig. 2).

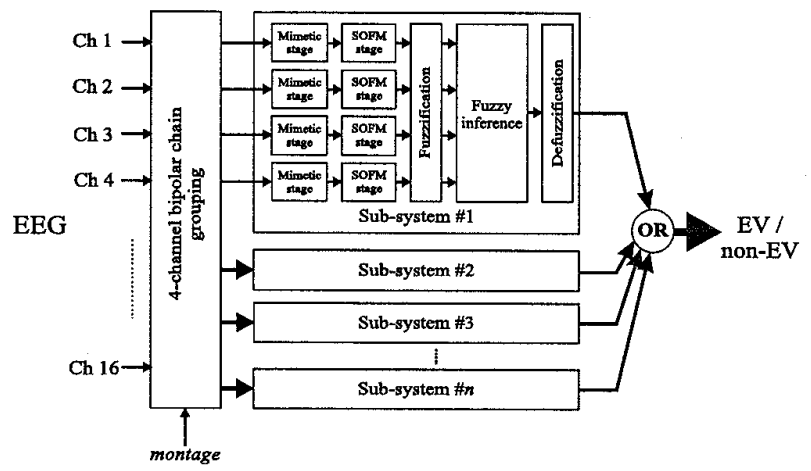

Fig. 2. The spatial-combiner in relation to the overall spike detection system.

The spatial-combiner combines the outputs of the SOFM stage in such a way as to confirm the presence of an EV on two or more channels of the EEG and, hence, report the detection of an EV. If the spatial pattern across the outputs of the previous stage is inconsistent with the presence of an $\mathrm{EV}$, it is rejected. In essence, the spatial-combiner uses a number of rules which specify allowable combinations of individual (i.e., single-channel) EDs across channels to detect the presence of an EV. The spatial-combiner works on a 4channel bipolar electrode chain basis, where the incoming (bipolar) EEG is examined based on identical sub-systems which group 4-channel bipolar chains together according to the current bipolar montage in use. The combiner relies on two pieces of information for each bipolar chain: (a) a probability value assigned to each candidate-ED (CED) on each channel by the SOFM stage and (b) the polarity of each CED within the bipolar chain.

The crisp inputs to the spatial-combiner (i.e., the probabilities of true ED as output by each SOFM of the previous stage) are fuzzified by using the fuzzy sets defined in Fig 3a. The fuzzy sets are defined to be: NB (negative big), NS (negative small), ZE (zero), PS (positive small) and $\mathrm{PB}$ (positive big). Each sub-system produces a single output 
which is defuzzified using the fuzzy sets described in Fig. $3 \mathrm{~b}$. The 4 fuzzy sets are defined to be: ZE (zero), POS (possible), PRO (probable) and DEF (definite). In both cases trapezoidal membership functions are used because of their ease of implementation. The method of composite maximum was adopted for the defuzzification process such that the rule most representative of an 'allowable' EV distribution across the 4 inputs contributes to the fuzzy output set label and the membership value.

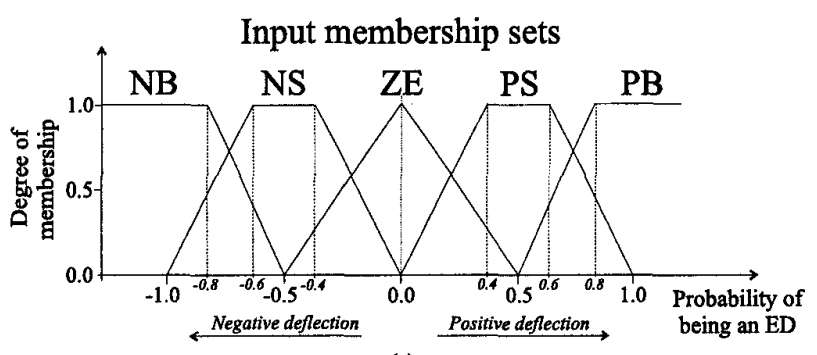

(a)

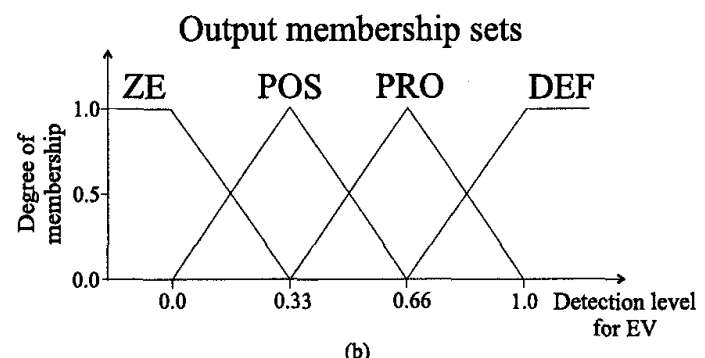

Fig. 3. The fuzzy sets used to define (a) the inputs to, and (b) the outputs of the spatial-combiner.

The fuzzy-rules are drawn-up based on our pre-defined knowledge of how an EV will manifest itself across a bipolar electrode chain. Each rule covers the possibility of a focal event at points along the bipolar electrode chain. As the probabilities assigned to each CED (by the SOFM stage) on each channel can take any value from 0 to 1 , it would take a great many rules to cover every combination of polarity and probability value for each possible EV focus along a 4 channel chain. Through the use of fuzzy logic, the generation of the spatial rules becomes easier as no explicit mathematical models are needed that describe the underlying process. As there are now only 5 fuzzy (input) variables, for a 4 channel electrode chain there are a maximum of $5^{4}=625$ possible rules which cover all the possible combinations of inputs. However, a large number of these rules are meaningless and are therefore not used. This results in 127 distinct fuzzy rules describing allowable combinations of fuzzy variables for the 4 inputs of each sub-system. Each rule derived in this manner is assigned an outcome of either DEF, PRO or POS. Fig. 4 describes one such fuzzy-rule derived in the manner described here. (The actual fuzzy output label assigned to each particular rule was arbitrarily based on the number of $\mathrm{PB} / \mathrm{NB}$ and PS/NS variables assigned to each particular rule.) The detection of an EV can then be made (a) if the crisp output exceeds a given threshold or (b) if the fuzzy output is either of POS, PRO or DEF.

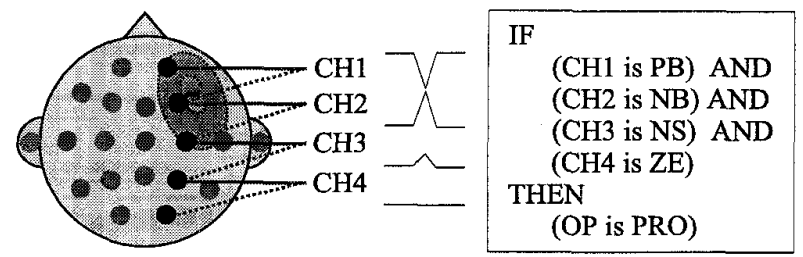

Fig. 4. An example fuzzy rule obtained in the instance of a focus at electrode 2 along a 4 channel (bipolar) chain of electrodes.

The underlying assumption when deriving the fuzzy spatial rules is that a focal EV is detected along the bipolar chain of electrodes. Generalized EVs, however, show no distinct focus across a number of 4 channel bipolar chains but will appear as a focal event at the end (or beginning) of each chain. Thus, the rules derived using the above method are able to detect generalized activity.

\section{Subjects}

The EEG was recorded by scalp electrodes placed according to the International 10-20 system. Sixteen channels of EEG were recorded simultaneously for bipolar montages only. The amplified EEG was bandpass filtered between 0.5 and $70 \mathrm{~Hz}$ using a five-pole analog Butterworth filter, sampled at $200 \mathrm{~Hz}$ and digitized to 12 bits. All data were stored for later off-line processing.

The performance of the system was tested with 7 EEGs containing epileptiform activity ( $4 \mathrm{x}$ generalized, $2 \mathrm{x}$ multifocal, and $1 \mathrm{x}$ focal as graded by 2 or 3 EEGers) and 1 normal EEG (i.e., no epileptiform activity as graded by all 3 EEGers). All of the EEGs contained significant artifact such as widespread EMG, persistent electrode movement and bursts of alpha-waves and no segment was discarded because of excessive artifact.

\section{Performance}

The test-set EEGs were presented to the multi-stage system trained in a similar manner to that of [8] with 35 different training set EEGs. Measures of sensitivity, selectivity and false detections/hour were calculated for each of the test-set EEGs, (a) at the output of the SOFM stage (i.e., before spatial combination) and (b) at the output of the spatial combiners, in order to assess the performance of spatial analysis in the spike detection process. 


\section{RESULTS}

At the output of the SOFM stage the overall sensitivity was measured at $56.6 \%$ with a corresponding selectivity of $8.6 \%$ and a false detection rate of $359 /$ hour. (A detection was assumed at the SOFM stage if any channel put forward a probability of greater than 0.8 ). The performance at the spatial-combiner stage was measured at a sensitivity of $55.3 \%$ and a selectivity of $82.0 \%$ coupled with a false detection rate of 7.2 false detections/hour. A detection was counted every time the spatial-combiner stage output a fuzzy label of either POS, PRO or DEF on any 4 channel sub-system.

\section{DISCUSSION}

The detection threshold of 0.80 at the SOFM stage was chosen so that the sensitivities at both the SOFM stage and the following spatial stage were similar, allowing a direct comparison between the selectivities and false detection rates. The detection threshold has no bearing on the performance of the overall system but is used here simply to obtain a measure of performance before the spatial-combiner stage. A sensitivity of $56.6 \%$ at the SOFM stage is reasonable but a false detection rate of $359 /$ hour is unacceptably high approximately 1 false detection every $10 \mathrm{~s}$ of EEG. (A lower detection threshold at the SOFM stage could, of course, give a higher sensitivity for this stage, but at the expense of a lower selectivity and much higher false detection rate). This notwithstanding, the performance at the SOFM stage indicates that a large quantity of the CEDs were assigned a reasonably high probability by each channel SOFM based on their single channel morphology.

The performance at the spatial-combiner stage indicates a massive increase in the selectivity from $8.6 \%$ to $82.0 \%$ coupled with an equally impressive 7.2 false detections/hour - one false detection every 8.3 minutes of EEG. This false detection rate reflects a 50 -fold reduction in rate from the SOFM stage. Of particular note are the results of the normal EEG. For this EEG the SOFM stage resulted in a high false detection rate of $302 /$ hour. Following spatial analysis, all false detections were eliminated. These results can only support our contention of the crucial importance of spatial analysis in the spike detection process.

Through the use of fuzzy logic, an approximation to the spatial reasoning used by EEGers in the spatial combination of the single channel EEG was possible. This avoids the need for exact mathematical models to represent the distribution of EVs across channels and maximizes the use of the probabilistic nature of the outputs from the single-channel SOFM stages. The fuzzy rules implemented were derived on our expectations of allowable combinations of single channel CEDs and resulted in the formation of a fuzzy rule base consisting of 127 such rules. The rules derived have performed quite well. Furthermore, the membership functions used were based on our expectations of the relative distributions of each member and although ad hoc resulted in equally acceptable results.

The immense gains to be obtained following the incorporation of spatial-contextual reasoning in the spike detection process have been more than proved with the results obtained.

\section{REFERENCES}

[1] J. Gotman, "Automatic recognition of interictal spikes", in Long term monitoring in epilepsy EEG supplement No. 37, J. Gotman, J.R. Ives, and P. Gloor, Eds., pp.99-114. Elsevier, 1985.

[2] A.A. Dingle, R.D. Jones, G.J. Carroll and W.R. Fright, "A multistage system to detect epileptiform activity in the EEG", IEEE Trans. Biomed. Eng., vol. 40, pp.1260-1268, 1993.

[3] J.R. Glover, N. Raghavan, P.Y. Ktonas and J.D. Frost, "Context-based automated detection of epileptogenic sharp transients in the EEG: Elimination of false positives", IEEE Trans. Biomed. Eng., vol. 36, pp.519-527, 1989.

[4] A.J. Gabor and M. Seyal, "Automated interictal EEG spike detection using artificial neural networks", Electroenceph. Clin. Neurophysiol, vol. 83, pp. 271-280, 1994.

[5] W.R.S. Webber, B. Litt, K. Wilson and R.P. Lesser, "Practical detection of epileptiform discharges (EDs) in the EEG using an artificial neural network: a comparison of raw and parameterized EEG data", Electroenceph. Clin. Neurophysiol., vol. 91, pp.194-204, 1994.

[6] R.D. Jones, A.A. Dingle, G.J. Carroll, R.D. Green, M. Black, I.M. Donaldson, P.J. Parkin, P.J. Bones and K.L. Burgess, "A system for detecting epileptiform discharges in the EEG: real-time operation and clinical trial", Proc. $18^{\text {th }}$ Ann. Int. Conf. IEEE EMBS, Amsterdam, 2 pages (CDROM), 1996.

[7] C.J. James, R.D. Jones, P.J. Bones and G.J. Carroll, “The self-organising feature map in the detection of epileptiform transients in the EEG", Proc. $18^{\text {th }}$ Ann. Int. Conf. IEEE EMBS, Amsterdam, 2 pages (CDROM), 1996.

[8] L.A. Zadeh, "Fuzzy sets", Inform. Contr., vol. 8, pp.338-353, 1965.

[9] G. Klir and T. Folger, "Fuzzy sets, uncertainty and information”, Prentice-Hall, Englewood Cliffs:NJ, 1988.

[10] H. Zimmermann, "Fuzzy sets, decision making and expert systems", Kluwer Academic Publishers, Boston, 1986.

[11] C.J. James, "The detection of epileptiform activity in the electroencephalogram using artificial neural networks", $P h D$ Thesis, University of Canterbury, Christchurch, New Zealand, 1997. 